Journal of

Synchrotron

Radiation

ISSN 0909-0495

Received 15 October 2004

Accepted 19 April 2005

(C) 2005 International Union of Crystallography

Printed in Great Britain - all rights reserved

\section{Nanoarchitectures of the animal extracellular matrix: opportunities for synchrotron radiation studies on collagen and fibrillin}

\author{
T. J. Wess ${ }^{a *}$ and D. E. Cairns ${ }^{b}$ \\ ${ }^{a}$ Biophysics Group, Department of Optometry and Vision Sciences, Cardiff University, Redwood \\ Building, King Edward VII Avenue, Cardiff CF10 3NB, Wales, UK, and ${ }^{\mathbf{b}}$ Department of Computing \\ Science and Mathematics, University of Stirling, Stirling FK9 4LA, Scotland, UK. \\ E-mail: wesstj@cf.ac.uk
}

\begin{abstract}
The extracellular matrix comprises structures that support the architectural organization of virtually all animal tissues. Within this architecture, two classes of protein assemblies found as long slender fibrils (collagen and fibrillin) characterize the bulk of the extracellular matrix. In both classes of fibrous protein, the molecular organization within a fibril ensures that the properties of the individual molecules transcend to the nanostructural and mesoscopic levels of structural organization and thence the tissue itself. The composition of the fibrils, in conjunction with other biomolecules and their suprafibrillar architecture, facilitates the formation of tissues as diverse as skin, tendon, cornea ciliary zonules and aorta. Here the relative tear resistance, strength, transparency and optical properties are paramount for proper function. Many structural investigations of fibrous protein structure have relied heavily on the use of synchrotron radiation in order to elucidate molecular packing, primarily due to the distinct benefits that X-ray diffraction provides, such as minimal sample preparation, rapid data collection and in situ mechanical testing. In this paper, an overview of the investigations that have revealed different levels of molecular architecture in fibril-based tissues is presented. Emerging future technology and how this can be matched with the pressing questions in extracellular matrix biology are also discussed.
\end{abstract}

Keywords: collagen; fibril; fibrillin; structure; X-rays.

\section{Introduction}

Fibrous proteins are by far the most abundant proteins in animals. The ability of molecular connections within a fibre to span a cell or indeed a tissue is explicit in the contractile function of muscle, the tensile function of tendon and the elastic function of the aorta. The extracellular matrix of animals is comprised of a series of molecular composites where fibrous proteins such as collagen, elastic proteins such as elastin, fibrillin and keratin have a major role. The association of monomeric protein structures and their interaction within a fibrillar structure is of central importance in understanding the structure-function relationship within extracellular matrix-rich tissues. There is a balance between a crystalline organization directed by specific intermolecular interactions and liquid-like interactions that evolve from less specific associations characteristic of liquid-crystalline behaviour. Both are important in the overall mechanical properties of the tissue.
Collagen is the most abundant protein in animals, and provides the main organic structural component of tissues such as skin, tendon, bone, cartilage and cornea. Collagen is characterized as a triple-helical molecular rope, and this organization is employed for its strength (fibrillar structures), its ability to facilitate spatial organization of globular domains (C1q and acetylcholine esterase) or both functions (basement membrane) (Hulmes, 2002). By weight, the majority of collagen found in animals is in a fibrillar form (Kadler, 1995; Brodsky \& Eikenberry, 1982), where the supramolecular organization of the triple helices ensures that the molecular strength transcends the higher levels of organization to the tissue level. Collagen in the fibrillar form self-organizes into a series of architectural levels, some more clearly characterized than others.

Fibrillin is a ubiquitous gene product expressed in many matrix tissues and is especially abundant in tissues that exhibit long-range elastic recoil (Sherratt et al., 2001). Fibrillin is usually found as the central component of fibrillin-rich 
microfibrils (MFs) which are essential parts of most developing and mature connective tissues in all animal phyla, but exhibit clearly different functions depending on the tissue studied. They are found in force-bearing tissues such as tendon, periodontal ligament or zonular fibres (suspensory ligaments) of the lens, but also in the arteries of the invertebrate, implying that MFs account for the elasticity of the highly developed circulatory systems of invertebrates. Fibrillin occurs as three forms, fibrillin 1-3 (Quondametteo et al., 2002). The exact role of fibrillin 3 remains undefined whereas fibrillin 2 has a preferential interaction with elastin and fibrillin 1 to form supramolecular structures that are not elaborated by elastin deposition (Handford, 2000). All are large glycoproteins $(350 \mathrm{kDa})$ and possess multidomain structures. The individual molecules have an extended rigid conformation and are approximately $160 \mathrm{~nm}$ in length.

The similarity (and differences) in structural features of fibrillin and collagen-based fibrillar structures deserves comparison. Indeed, the intimate relationship between their properties within all matrix tissues was revealed when the condition Marfan's syndrome, a genetic pathology associated with hyperextensibility, dislocation of the eye lens and aortic aneuryism, was for almost 100 years ascribed to a collagen defect but was finally mapped to a defect in the fibrillin 1 gene (Kielty \& Shuttleworth, 1995).

In this article we describe the current structural knowledge of these two important fibrillar components of the extracellular matrix, from the collagen helix to the suprafibrillar organization that confers the unique biophysical properties of each tissue.

\section{Axial structure}

The molecular organization of collagen and fibrillin lends itself to investigations based on diffraction and microscopy. Collagen fibrils are easily recognized in electron microscopy as banded $\sim 67 \mathrm{~nm}(D)$ repeating structures. The banding corresponding to almost equal regions of high and low electron density produces a projection corresponding to a step function. Such a repeating structure can be accounted for by the relative stagger of individual $300 \mathrm{~nm}$ collagen molecules by $67 \mathrm{~nm}$ or integer multiples thereof. Since the length of the collagen molecule approximates to $4.4 \times D$ segments, the alignment must lead to one segment of the collagen molecule contributing only $0.4 \mathrm{D}$ of collagen helix. The combination of molecules in this simple (Hodge \& Petruska, 1963) scheme has been at the core of understanding axial interactions for over 40 years. In projection the $67 \mathrm{~nm}$ repeat contains the regions of high density (or overlap regions) which correspond to positions where all collagen molecules make a full contribution to the structure; the less-dense gap region corresponds to the part of the projection where the collagen segment is missing. This is shown in Fig. 1.

The X-ray fibre diagram of collagen is dominated by the contrast of the gap and overlap region where the sharpness and extent of the meridional series correspond to the axially projected electron density. Up to 140 meridional diffraction intensities have been observed corresponding to a resolution of $0.5 \mathrm{~nm}$, and a detailed electron density map of the projected structure has been made by Orgel et al. (2000). This shows the collagen structure to be stabilized principally by the interaction between charged regions on adjacent collagen segments. The $\mathrm{C}$ terminal contains a 25 amino acid non-helical telopeptide that in the above study was shown to be folded, confirming earlier models. Although the major features of collagen axial packing are now well resolved, there remain structural features that require further investigation; two of these are the variability of the axial rise per residue (Paterlini et al., 1995) and the detailed structure of the non-helical telopeptide regions (Jones \& Miller, 1991).

\section{Axial structure of fibrillin}

Fibrillin presents similarities to collagen fibrils in as much that it contains a long-range $(56 \mathrm{~nm})$ axial structure which appears to be a feature of fibrillin microfibrils in all animal phyla. Here the repeating regions of high and low density are termed the bead and interbead region reflective of the 'beads on a string' appearance in early electron micrographs. These can clearly be seen in Fig. 2. The basis for axial density contrast within fibrillin microfibrils is not resolved. The molecular stagger or folding of fibrillin molecules may provide the basis for overlapping molecules and contrast variation. However, there is strong evidence that fibrillin microfibril-associated proteins attach at regular intervals to the underlying fibrillin architecture. The X-ray diffraction meridional series of intensity from fibrillin microfibrils (Fig. 3) only extends to eight or, at best, ten orders of diffraction; these reflections are relatively weak compared with the collagen meridional series and their inherent breadth in both axial and lateral directions indicates a lower degree of crystallinity or dispersion into smaller diffracting units. An interesting feature of the meridional series is the presence of strong third and sixth orders of diffraction that are not easily accounted for by the lack of

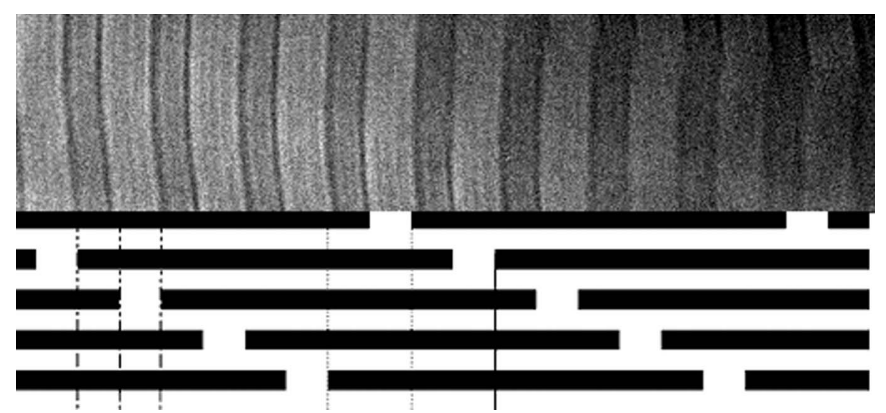

Figure 1

The axial organization of collagen molecules in a collagen fibril and the pattern this arrangement produces, as revealed by X-ray diffraction and electron microscopy. The individual $300 \mathrm{~nm}$-long collagen molecules are axially aligned in the fibril according to the Hodge \& Petruska (1963) model. This produces the gap-overlap step function of electron density that underlies the meridional series of reflections in the fibre diagram and also produces the characteristic banding pattern of $67 \mathrm{~nm}$ seen in electron micrographs of collagen fibrils. 


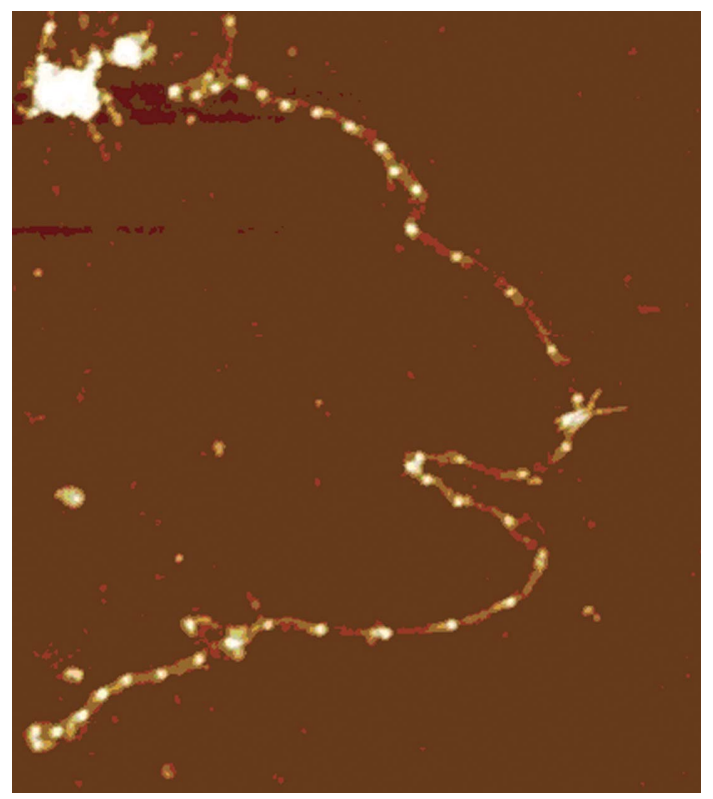

Figure 2

The variability in the beaded structure of fibrillin-rich microfibrils is clearly demonstrated here. Adult bovine ciliary zonule microfibrils (extracted/purified by bacterial collagenase/size exclusion) were adsorbed on mica coated with poly L-lysine and subjected to molecular combing in water, followed by electron microscopy. A $56 \mathrm{~nm}$ repeating beaded structure is clearly visible at the top of the figure, which represents the relaxed molecular state. Extension of the periodicity up to $120 \mathrm{~nm}$ is clearly visible in the centre of the same microfibril. The figure was kindly donated by $M$. Sherratt and C. Kielty, University of Manchester.
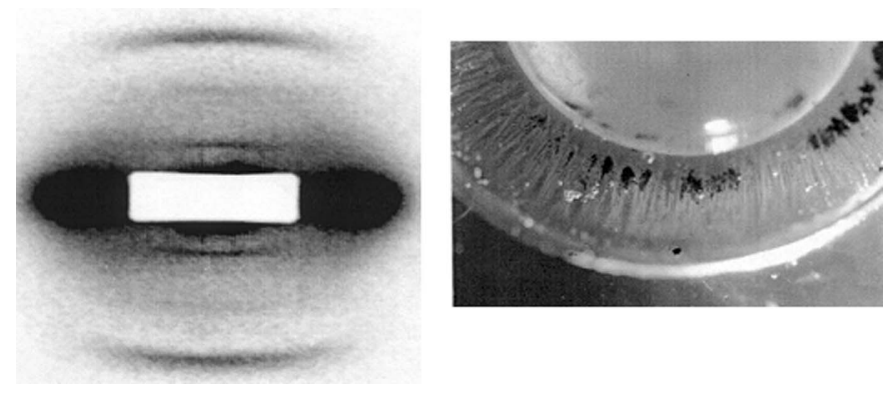

Figure 3

X-ray diffraction pattern (left) of fibrillin from bovine zonular filaments (right) recorded at beamline 2.1 of the Daresbury synchrotron. The first three orders of the $1 / 56 \mathrm{~nm}$ repeat meridional diffraction are shown here: the predominant third diffraction order indicates suprafibrillar packing (see text), the strong equatorial reflection corresponds to the side-by-side interactions of the fibrillin microfibrils. The figure on the left is reproduced with permission by Rockefeller University Press from Wess, Purslow, Sherratt et al. (1998). The figure on the right shows an enucleated bovine eye lens surrounded by ciliary zonules.

structural features with these periodicities within the axially projected structure.

\section{Lateral packing in fibrillar collagens}

While the basis for axial packing of collagen molecules has been well resolved, an understanding of lateral packing is still limited. Investigation of lateral packing has focused on fibrillar structures that exhibit long-range crystallinity, such as those from rat-tail tendon (North et al., 1954; Wess, Hammersley et al., 1998). Detailed investigations of packing modalities within fibrils from rat-tail tendon favour the presence of microfibrillar structural units arranged as compressed microfibrils on a triclinic unit-cell lattice (Fraser et al., 1983; Wess et al., 1995). Distortion of a regular pentagon to a compressed structure that allows a quasi-hexagonal lateral packing (Trus \& Piez, 1980) gives a more realistic packing density whilst still adhering to the pentameric molecular connectivity (topology) as suggested by Smith (1968). The detail of these structural units has been revealed recently by structure determination of the electron density map of a single collagen unit cell by X-ray diffraction using adapted protocols from the multiple isomorphous replacement techniques of phase determination in macromolecular crystallography (Orgel et al., 2001).

These studies concur in identifying a one-dimensional staggered microfibrillar structure with intermicrofibrillar crosslinks. The crosslinks are significant at the supramicrofibrillar level of hierarchical connectivity and also provide an explanation for the difficulty in isolating individual microfibrils. Although there is evidence for specificity of molecular packing indicated by crystallinity, a significant amount of diffuse scatter is also present in the X-ray fibre diagram of fibrillar collagen, indicating a large amount of static or even dynamic disorder within fibrils. Where fibrils contain crystalline regions, significant levels of collagen molecules exhibiting liquid-like disorder are also believed to be present, with the lower-density gap region of the fibril structure thought to be more disordered than the overlap (Fraser et al., 1987; Wess, Hammersely et al., 1998), such that the molecular packing of collagen has been compared with that of a liquid or liquidcrystalline-type packing, where only local molecular interactions are of significance (Hukins \& Woodhead-Galloway, 1977; Fratzl et al., 1993). In X-ray diffraction of some fibril structures, the only observable feature is the scatter associated with a liquid-based packing (see Fig. 4). This would indicate that there are a number of levels of lateral molecular organization present in collagen fibrils spanning liquid to crystalline states.

Definition is needed of the relationship of compatibility of crystallinity, disorder and supramolecular topology of crystalline packing within a fibril. Electron micrograph studies of lateral sections of fibrils have observed the long-range crystalline structure resulting from organized lateral packing (Hulmes et al., 1985), and an assessment of the compatibility of the many proposed fibrillar packing models emerging with electron micrograph and X-ray diffraction data has also been attempted (Hulmes et al., 1995). Ordered crystalline to disordered liquid-like structures were modelled and their corresponding Fourier transforms compared with the diffraction pattern of tendon. All of the models were either unable to accommodate both crystallinity and disorder to the appropriate extent or contained unrepresentative molecular correlations. The best agreement with the diffraction data was found with a model based on concentric rings of microfibrillar structures, developed within this particular study. The proposed model comprised sectors of crystalline order 


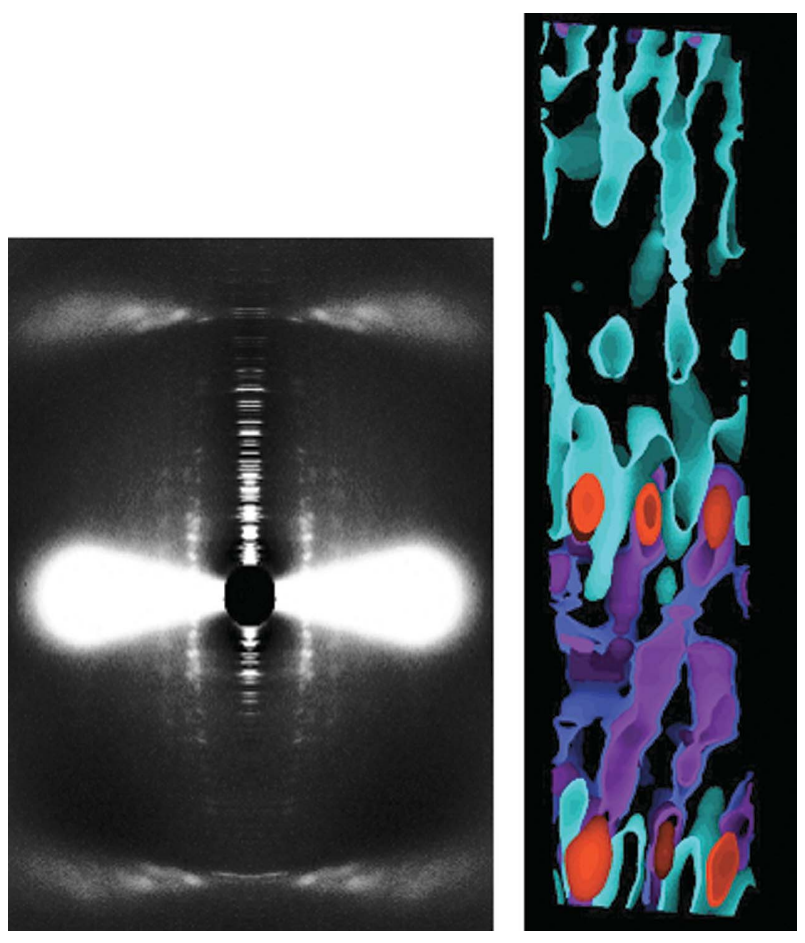

Figure 4

Left: X-ray diffraction pattern of collagen fibrils in rat-tail tendon. Reproduced with permission by Elsevier Press from Orgel et al. (2000). The X-ray diffraction pattern of rat-tail tendon contains a series of intense meridional reflections corresponding to axial molecular organization. Three-dimensional microcrystalline domains of collagen molecules produce discrete Bragg peaks in the equatorial $(m=0, n=0)$ helix layer plane and parallel to the meridian of the diffraction pattern. The resolution of the diffraction data extends to approximately $1.0 \mathrm{~nm}$ along the equator and $0.54 \mathrm{~nm}$ parallel to the meridian. Elements of diffraction can also be seen corresponding to the $(m=0, n=-1)$ helical layer line. Such diffraction images were used to produce the electron density maps of collagen structure shown on the right. This is reproduced with permission by Elsevier Press from Orgel et al. (2001). Here the higherdensity overlap region (bottom) can be seen to contain five molecular segments whereas the less easily visible gap region contains more diffuse density although some evidence of molecular paths can be seen. The gap region is likely to contribute to the liquid-like order in the collagen fibril. The vertical aspect represents the $67 \mathrm{~nm}$ periodicity and this has been compressed by a factor of eight so that the molecular paths are exaggerated. The horizontal scale is $5 \mathrm{~nm}$.

together with disordered grain boundaries. This structure enabled crystallites to be maintained uniformly within a fibril yet exhibit a circular cross section. This structure provides a potential explanation for the curvature of the crystallites as reported by Fraser et al. (1983). Furthermore, an understanding of the structural requirements required for growth can be obtained, with incremental deposition of microfibrils occurring at the fibrillar surface. This correlates with the $8 \mathrm{~nm}$ quantal variation in fibril diameter that was noted by Parry \& Craig (1979). Fig. 5 provides a cross section of the suggested fibrillar structure.

\section{Lateral packing in fibrillin microfibrils}

The molecular packing within a fibrillin microfibril probably has a greater degree of similarity with a collagen microfibril

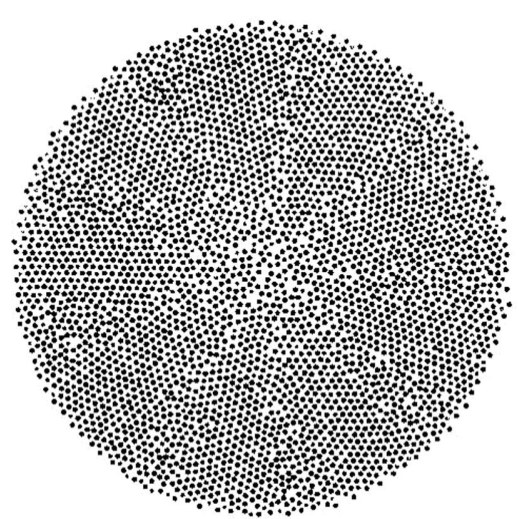

Figure 5

The relaxed radially packed cylindrical structure of molecular segments in the overlap region of a fibril model. Proposed cross-sectional structure of a collagen fibril (with a $50 \mathrm{~nm}$ radius) consisting of $\sim 4000$ collagen molecules where each dot represents a collagen triplex. The molecular arrangement is based on concentric rings of microfibrils and then energy minimized. Crystalline regions are defined by disordered grain boundaries; the fibril core is relatively poorly ordered. The structure therefore contains regions of static disorder as well as the disorder that is present in the gap region. The size of a fibril may well govern the amount of overall crystallinity present. Modified from Hulmes (2002) with permission from Elsevier Press.

than a collagen fibril. Here the fundamental difference between packing of relatively similar linear helical molecular motifs is contrasted with the fibrillin molecule where the overriding feature of an individual molecule is the multiglobular domain structure (Haston et al., 2003). This is possibly the point at which trying to draw an analogy between collagen and fibrillin becomes invalid. However, the similarity between fibrillar structures at other levels justifies the overall comparison. There is currently no consensus of opinion on the lateral packing of fibrillin molecules within an individual fibrillin microfibril (Handford et al., 2000; Baldock et al., 2001; Downing et al., 1996; Qian \& Glanville, 1997; Trask et al., 1999; Reinhardt et al., 1996). Indeed, the number of molecules contributing to the lateral structure of a microfibril remains unresolved but probably corresponds to one to three molecules. The estimation of the lateral size of a fibrillin microfibril has been difficult to ascertain since the characteristic beaded filament with $10-12 \mathrm{~nm}$ diameter as described by electron microscopy appears to give a scattering function that corresponds to a molecular diameter of more than $25 \mathrm{~nm}$ in resting intact tissue.

\section{Suprafibrillar structures}

One of the more neglected fields of research for both collagen and fibrillin is the detailed analysis and basis for the supramolecular architectures made by fibrils. This level of association is critical to the link between fibrillar integrity and functional tissues. The organization of fibrils in specific uniaxial, planar, helical and isotropic architectures is often the modulating factor in the overall characteristic of a tissue. 


\section{Suprafibrillar structures of collagen}

The mesoscopic organization of fibrillar structures is critical to the final function of a tissue. Cartilage, skin, cornea and tendon each serve as examples of where suprafibrillar collagen architecture provides the major characteristics of the macroscopic tissue. In cartilage, the superficial layer of many articulating joints is a two-dimensional mesh that covers a more disordered fibrillar network. These arrangements spread the force received at a joint and provide a smooth articulating surface (Wilkinson \& Hukins, 1999). In skin, the suprafibrillar architecture is that of a two-dimensional felt that provides biaxial mechanical strength and strong tear-resistant properties (Purslow et al., 1998). In tendon, the preferred uniaxial arrangement of fibrils allows the optimal transmission of force from muscle to bone to allow movement. In cornea, a specific organization of fibrils with a highly regulated diameter results in a three-dimensional architecture of orthogonally arranged sheets (Meek \& Quantock, 2001); this is essential for the transparency and strength of the tissue. Many collagen-based architectures can be probed with X-ray diffraction that allows determination of molecular organization in the hydrated state. A common theme of collagen supramolecular structure is the presence of architectures that resemble cholesteric and precholesteric liquid-crystal arrangements (Martin et al., 2000). The precise molecular mechanisms that determine the three-dimensional architectures of tissues are largely unknown, although suggestions of long-range interactions from liquid-crystal arrangements of collagen structures may provide a clue.

\section{Suprafibrillar structures of fibrillin}

Fibrillin structures have long been recognized in electron micrographs as ubiquitous components of the extracellular matrix. They form bundles and thence architectures that accommodate the compliance of a tissue and often are coaligned with bundles of collagen fibrils. A basis for the above fibrillar organization was identified more recently by X-ray diffraction, where the specific $18 \mathrm{~nm}$ relative staggering of microfibrils was shown to be found in fibrillin-rich tissues such as ciliary zonules of the eye (Wess, Purslow \& Kielty, 1998). Such staggering of microfibrils may allow the energy stored within the elastic deformation of individual microfibrils to be transferred to higher levels of architecture. An electron micrograph showing staggered fibrillin molecules is shown in Fig. 6.

\section{Tensile properties of fibrillar collagen-based tissues}

The collagen extracellular matrix provides the framework that is responsible for withstanding or transferring tensile loads. Taken as a whole, the interactions of the architecture of the collagen molecules, micro-fibrils and fibres produces the specific properties of collagen (Puxkandl et al., 2002). Structures such as tendon, which are constituted mainly from collagen fibrils, are frequently used as a source of material for gaining an insight into collagen mechanics. The predominant technique that has been used in order to gain an understanding of the molecular principles for deformation of fibril structures is X-ray diffraction. This is primarily because it allows for the deformation to be effected under experimental conditions, yet still enables a diffraction image to be obtained.

The principle effects of strain in collagen-based tissue are molecular elongation and molecular shear inside a fibril together with the shear that occurs with the deformation of the proteoglycan-rich matrix between fibrils. It follows from this that the above molecular organization and crosslinking between these fibrils should be appropriately formed. The outcome of exposing fibres to strain is highly dependent on the rate at which the strain is applied. High initial strain rates will produce elongation of the collagen molecules and begin to cause further shear effects within a fibril. In contrast, a slow rate of strain will cause a shear to occur in the matrix between fibrils and will also produce creep (Sasaki et al., 1999).

The study of fibril behaviour in tissues using synchrotron radiation has been crucial to gaining an understanding of structural alterations because this approach provides the facility to monitor transient structural features within realistic time frames. Experiments studying the effects of stress-strain carried out in synchrony with X-ray diffraction have shown that only $40 \%$ of the increase in the $D$ period that occurs before failure occurs due to extension of the collagen helix (where the molecule extends from $67 \mathrm{~nm}$ to $69 \mathrm{~nm}$ ). It has been concluded that the remaining extension has to occur due to molecular rearrangement. This effect can be observed as a

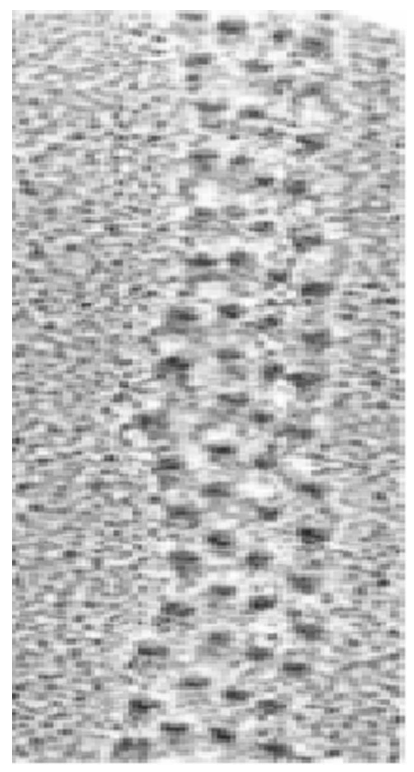

Figure 6

Electron micrograph of suprafibrillar arrays adapted with permission by Rockefeller University Press from Wess, Purslow \& Kielty (1998). Here, in addition to the characteristic $56 \mathrm{~nm}$ periodicity that can be observed in rotary shadowed microfibrils, there is evidence of specific interfibrillar interactions. The specific $D / 3$ staggered relationship between beaded fibrillin microfibrils can be seen in a rotary shadowed aggregate. X-ray diffraction evidence points to far more of such associations occurring in the intact tissue since the third and sixth meridional orders of diffraction on a $56 \mathrm{~nm}$ lattice are predominant. 
shift in the relative intensity observed for the second- and third-order Bragg peaks in meridional series. This shift indicates a significant distortion compared with the normally observed gap/overlap step function characteristic of fibrillar collagens (Sasaki \& Odajima, 1996). This type of in situ experiment has lead to the principle that a number of different processes are involved in responses to strain effects and that different processes will dominate according to the degree of strain. Under small loads, a straightening of the kinks in the collagen structure is observed (Fratzl et al., 1998), beginning at the fibrillar level and then at the molecular level. An increase in the strain levels is understood to produce molecular gliding within the fibrils and eventually leads to the breakdown of the fibril structure. Attempts to quantify the distortions produced by in situ strain effects have only been partially successful. Three simple models for molecular elongation and collagen rearrangement within a fibril were proposed. The first model suggested alteration of the helical pitch, the second an increase in the gap region and the third proposed a slippage with respect to the laterally adjoining molecules. Such alterations in molecular structure can only account for certain features within the collagen fibre diagram that change with strain; a satisfactory fit with the $\mathrm{X}$-ray diffraction data is still sought.

\section{Elastic properties of fibrillin-rich tissues}

Fibrillin is usually described as an elastic protein. This is substantiated by the fact that fibrillin is a core structure within elastic fibres, and individual microfibrils have been observed with a variety of bead-to-interbead spacings that may provide the molecular basis for elasticity. The elastic recoil of the bead-interbead spacing was proven by X-ray diffraction (Wess, Purslow \& Kielty, 1998) where the meridional peak spacing was shown to increase from a 1/56 lattice to a $1 / 80 \mathrm{~nm}$ lattice. Subsequent studies showed that this was reversible at extensions of up to $100 \mathrm{~nm}$ with plastic deformations occurring over 110-120 nm (Haston et al., 2003).

The stress-strain curve exhibits similar characteristics to collagen where the initial toe region corresponds to the realignment of the microfibril bundles followed by a linear regime of deformation within the microfibril. More recent studies may indicate that the physiological function of fibrillinrich tissues may not require to access the bead deformation region of the stress-strain curve and the tissue may act as a stiff rod reinforcing a tissue (Sherratt et al., 2003). However, it has been difficult to ascertain the pretension associated with samples in vivo.

\section{The way forward}

A new approach towards solving some of the long-standing problems discussed above, e.g. in collagen structure, pathology and in the development of smart biomimetic materials, is the novel application of both newly emergent and well established techniques. Microfocus XRD, cryo-electron microscopy, atomic force microscopy and environmental scanning electron microscopy can each yield insights into the structural basis of collagen, while cryo-XRD inelastic neutron scattering and $\mathrm{X}$-ray photon correlation spectroscopy can be used to investigate molecular dynamics. These novel applications are particularly relevant to the study of tissues mainly composed of one collagen type, e.g. corneal stroma, tendon and skin (all primarily type I), where fibril diameter and packing are closely linked to properties and function.

Microfocus X-ray beams and the advent of nanofocus beams may allow the variation in structural features to be mapped along the length of a single collagen fibril. This may indicate that the crystalline packing only resides in the shaft region and the pointed tips may present different modes of lateral packing. It may be possible to conduct skin-core experiments on individual large (micrometre-diameter) collagen fibrils in order to probe depth-dependent difference in internal crystallinity. The contribution of individual molecular species to tissues that are composites of collagen and fibrillin-based structures will allow a more detailed relationship within composites to be defined. Speculative suggestions that fibrillin may form regions of microcrystallites based on the specific association of microfibrils may be substantiated by detailed nanobeam mapping of thin tissue sections.

Although many years of studies have shown the important interactions of collagen and fibrillin with cells, the overriding property of these tissues remains a mechanical one, therefore the opportunity to understand the structural/mechanical function relationship of these molecules is all important. The changes in length scale over which the mechanical deformation of tissue such as tendon or skin occur requires use of rapid data collection for high-, low- and even ultra-low-angle X-ray diffraction data. Detailed multidisciplinary studies of the deformation of collagen and fibrillin combined with the development of sophisticated models of the molecular behaviour are required to gain a much fuller understanding of these two proteins that are ubiquitous in animal life.

We wish to acknowledge the staff at ID02 and ID13 at ESRF in Grenoble and the staff at stations 2.1 and 14.1, SRS, Daresbury for all their help in data collection and analysis.

\section{References}

Baldock, C., Koster, A. J., Ziese, U., Rock, M. J., Sherratt, M. J., Kadler, K. E., Shuttleworth, C. A. \& Kielty, C. M. (2001). J. Cell. Biol. 152, 1045-1056.

Brodsky, B. \& Eikenberry, E. F. (1982). Methods Enzymol. 82, $127-$ 174.

Downing, A. K., Knott, V., Werner, J. M., Cardy, C. M., Campbell, I. D. \& Handford, P. A. (1996). Cell, 85, 597-605.

Fraser, R. D. B., MacRae, T. P. \& Miller, A. (1987). J. Mol. Biol. 193, 115-125.

Fraser, R. D. B., MacRae, T. P., Miller, A. \& Suzuki, E. (1983). J. Mol. Biol. 167, 497-521.

Fratzl, P., Fratzl-Zelman, N. \& Klaushofer, K. (1993). Biophys. J. 64, 260-266.

Fratzl, P., Misof, K., Zizak, I., Rapp, G., Amenitsch, H. \& Bernstorff, S. (1998). J. Struct. Biol. 122, 119-122.

Handford, P. A. (2000). Biochim. Biophys. Acta Mol. Cell Res. 1498, 84-90. 
Handford, P. A., Downing, A. K., Reinhardt, D. P. \& Sakai, L. Y. (2000). Matrix Biol. 19, 457-470.

Haston, J. L., Engelsen, S. B., Roessle, M., Clarkson, J., Blanch, E. W., Baldock, C., Kielty, C. M. \& Wess, T. J. (2003). J. Biol. Chem. 278, 41189-41197.

Hodge, A. J. \& Petruska, J. A. (1963). Aspects of Protein Structure, edited by G. N. Ramachandran, pp. 289-300. New York: Academic Press.

Hukins, D. W. L. \& Woodhead-Galloway, J. (1977). Mol. Cryst. Liq. Cryst. 41, 33-39.

Hulmes, D. J. S. (2002). J. Struct. Biol. 137, 2-10.

Hulmes, D. J. S., Holmes, D. F. \& Cummings, C. (1985). J. Mol. Biol. 184, 473-477.

Hulmes, D. J. S., Wess, T. J., Prockop, D. J. \& Fratzl, P. (1995). Biophys. J. 68, 1661-1670.

Jones, E. Y. \& Miller, A. (1991). J. Mol. Biol. 218, 209-219.

Kadler, K. (1995). Extracellular Matrix 1: Fibril Forming Collagens, Protein Profile 2, edited by P. Sheterline, pp. 491-619. Oxford: Academic Press.

Kielty, C. M. \& Shuttleworth, C. A. (1995). Int. J. Biochem. Cell Biol. 27, 747-760.

Martin, R., Farjanel, J., Eichenberger, D., Colige, A., Kessler, E., Hulmes, D. J. \& Giraud-Guille, M. M. (2000). J. Mol. Biol. 301, 1117.

Meek, K. M. \& Quantock, A. J. (2001). Prog. Retin. Eye Res. 20, 95137.

North, A. C. T., Cowan, P. M. \& Randall, J. T. (1954). Nature (London), 174, 1142-1143.

Orgel, J. P. R., Miller, A., Irving, T. C., Fischetti, R. F., Hammersley, A. P. \& Wess T. J. (2001). Structure, 9, 1061-1069.

Orgel, J. P. R., Wess, T. J. \& Miller, A. (2000). Structure, 8, 137-142.

Parry, D. A. \& Craig, A. S., (1979). Nature (London), 282, 213-225.

Paterlini, M. G., Nemethy, G. \& Scheraga, H. A. (1995). Biopolymers, 35, 607-619.
Purslow, P. P., Wess, T. J. \& Hukins, D. W. L. (1998). J. Exp. Biol. 201, 135-142.

Puxkandl, R., Zizak, I., Paris, O., Keckes, J., Tesch, W., Bernstorff, S., Purslow, P. \& Fratzl, P. (2002). Philos. Trans. R. Soc. Lond. B, 357, 191-197.

Qian, R.-Q. \& Glanville, R. W. (1997). Biochemistry, 36, 1584115847.

Quondamatteo, F., Reinhardt, D. P., Charbonneau, N. L., Pophal, G., Sakai, L. Y. \& Herken, R. (2002). Matrix Biol. 21, 637-646.

Reinhardt, D. P., Keene, D. R., Corson, G. M., Poschl, E., Bachinger, H. P., Gambee, J. E. \& Sakai, L. Y. (1996). J. Mol. Biol. 258, 104116.

Sasaki, N. \& Odajima, S. (1996). J. Biomech. 29, 1131-1136.

Sasaki, N., Shukunami, N., Matsushima, N. \& Izumi, Y. (1999). J. Biomech. 32, 285-292.

Sherratt, M. J., Baldock, C., Haston, J. L., Holmes, D. F., Jones, C. J. P., Shuttleworth, A., Wess, T. J. \& Kielty, C. M. (2003). J. Mol. Biol. 332, 183-193.

Sherratt, M. J., Wess, T. J., Baldock, C., Ashworth, J., Purslow, P. P., Shuttleworth, C. A. \& Kielty, C. M. (2001). Micron, 32, 185-200.

Smith, J. W. (1968). Nature (London), 219, 157-158.

Trask, T. M., Ritty, T. M., Broekelmann, T., Tisdale, C. \& Mecham, R. P. (1999). Biochem. J. 340, 693-701.

Trus, B. L. \& Piez, K. A. (1980). Nature (London), 286, 300-301.

Wess, T. J., Hammersley, A. P., Wess, L. \& Miller, A. (1995). J. Mol. Biol. 248, 487-493.

Wess, T. J., Hammersley, A. P., Wess, L. \& Miller, A. (1998). J. Mol. Biol. 275, 255-267.

Wess, T. J., Purslow, P. P. \& Kielty, C. M. (1998). J. Struct. Biol. 122, 123-127.

Wess, T. J., Purslow, P. P., Sherratt, M. J., Ashworth, J., Shuttleworth, C. A. \& Kielty, C. M. (1998). J. Cell Biol. 141, 829-837.

Wilkinson, S. J. \& Hukins, D. W. L. (1999). Radiat. Phys. Chem. 56, 197-204. 